\title{
Fluoroimmunoassay for Antigen Based on Fluorescence Resonance Energy Transfer Between Quantum Dots and Gold Nanoparticles
}

\author{
Can Wang ${ }^{1}$, Xiaogang You ${ }^{2}$, Hualin $\mathrm{Fu}^{2}$, Peng Huang ${ }^{1}$, Feng $\mathrm{Gao}^{2}$, Yunsheng $\mathrm{Chen}^{2}$, QuanDe Liao ${ }^{1 *}$ \\ ${ }^{1}$ Xiangya Hospital of Central South University, 87 Xiangya Road, Changsha410008, Hunan, P. R. China \\ 2.Institute of Nano Biomedicine and Engineering, Key Laboratory for Thin Film and Microfabrication Technology of the Ministry of Education, \\ Research Institute of Micro/Nano Science and Technology, Key Laboratory for the Genetics of Developmental \& Neuropsychiatric Disorders of \\ Ministry of Education, Bio-X Center, Shanghai JiaoTong University, Dongchuan Road 800, 200240 Shanghai, P. R. China \\ * Corresponding author: Q.D. Liao (qiandeliao@yahoo.cn)
}

\begin{abstract}
A unique, sensitive, and highly specific fluoroimmunoassay system for antigen detection using quantum dot and gold nanoparticles has been developed. The assay is based on the fluorescence quenching of quantum dots caused by gold nanoparticles coated with antibody. To demonstrate its analytical capabilities, the quantum dots were coated with anti-HBsAg monoclonal antibodies (QDs-MAb1) and gold nanoparticles coated with another anti-HBsAg monoclonal antibodies ( GNPs-MAb2) which specifically bound with HBsAg could sandwich the HBsAg captured by the immunoreactions. The sandwich-type immunocomplex was formed and the energy of quantum dots was transferred to gold nanoparticles as they were within a short distance, so that the fluorescence intensity of quantum dots was quenched. The fluorescence intensity of quantum dots at 570 nm was negative linear proportional to HBsAg concentration logarithm. The result showed that the limit of detection of the HBsAg was $0.928 \mathrm{ng} / \mathrm{mL}$. This new system can be extended to detect target molecules with matched antibodies and has broad potential applications in immunoassay and disease diagnosis.
\end{abstract}

Keywords: Antigen, Fluorescence resonance energy transfer, Fluoroimmunoassay, Gold Nanoparticles, Quantum Dots

Citation: C. Wang et al. Fluoroimmunoassay for Antigen Based on Fluorescence Resonance Energy Transfer Between Quantum Dots and Gold Nanoparticles. Nano Biomed. Eng. 2013, 5(3), 127-130.

DOI: 10.5101/nbe.v5i3.p127-130.

\section{Introduction}

Fluorescence resonance energy transfer (FRET) is a process whereby the electronic excitation energy of a donor chromophore is nonradiatively transferred to a nearby acceptor molecule via a through-space dipoledipole interaction between the donor-acceptor pair [1-5]. FRET occurs when there is appreciable overlap between the emission spectrum of the donor and the absorption spectrum of the acceptor. The strong distance dependence of the FRET efficiency has been widely exploited in studying the structure and dynamics of proteins and nucleic acids, in the detection and visualization of intermolecular association, and in the development of intermolecular binding assays [6]. FRET-based studies involving pairs of organic dye molecules as the donoracceptor complexes are often limited by cross-talk caused by spectral overlap of the donor and acceptor emission. The need for significant overlap between the emission and absorption spectra of the donor and acceptor, coupled with the narrow absorption spectrum of conventional organic dye molecules, makes it difficult to avoid direct excitation of the acceptor molecules at the excitation wavelength needed to efficiently excite the donor. In addition, the broad emission spectrum of the donor, with its long red tail, can often overlap significantly with the emission spectrum of the acceptor. Several recent reports have confirmed that luminescent semiconductor quantum dots (QDs), such as CdSe and CdTe, are able to participate in resonance energy transfer processes analogous to FRET [7-12], which makes these materials good candidates to overcome some of the limitations associated with conventional organic dye molecules in FRET-based studies of biomolecular structure, ligandreceptor binding, etc.

Semiconductor QDs are currently being investigated for their use as luminescent biological probes because of their high photostability relative to organic dye molecules and their unique, size tunable spectral properties [13-16]. 
In the past few years, QDs have been favorably adopted in the FRET-based studies due to their distinct optical characteristics [17]. In addition, gold nanoparticles (GNPs) have been of great interest because of their high extinction coefficient and a broad absorption spectrum in a visible light that is overlapped with the emission wavelength of usual energy donors [18-21]. As a photoluminescence (PL) quencher of organic dyes, GNPs were employed to detect single-mismatched nucleotides based on a molecular beacon technique and other protein interactions [17]. However, despite a number of bioanalytical applications of nanoparticles [22,23], to the best of our knowledge, there have been few attempts employing the PL quenching of QDs by GNPs for studying an immunoassay in aqueous solutions [24,25].

Herein we reported a novel immunoassay based on the modulation in FRET efficiency between QDs and GNPs in the presence of the HBsAg which sandwiched the interactions between QD- and GNPs-conjugated biomolecules. The antigen HBsAg was chose to prove the novel immunoassay method as a typical example.

\section{Experimental}

\subsection{Materials and reagents}

Bovine serum albumin (BSA), ethyl-3-(dimethylaminopropyl) carbodiimide(EDC), hydrogen tetrachloroaurate(III) trihydrate $\left(\mathrm{HAuCl}_{4}\right)$ and trisodium citrate were purchased from Shanghai Chemical Reagent Corporation (China). Hepatitis B Surface Antigen, antiHBsAg monoclonal antibodies (M701077, MAb2), antiHBsAg monoclonal antibodies(M701079, MAb1) were purchased from Fitzgerald Industries International, Inc. Water soluble carboxyl modified quantum dots of CdTe (QDs) were prepared by our laboratory [26]. All other chemicals were of analytical grade.

\subsection{QDs-MAb1 conjugate preparation}

The COOH-functionalized CdTe nanoparticles (QDs) were conjugated to anti-HBsAg monoclonal antibodies (MAb1) with the cross-linking reagent ethyl-3-(dimethylaminopropyl) carbodiimide. Briefly, $100 \mu \mathrm{L}$ of QDs (4 $\mathrm{mg} / \mathrm{mL})$ was incubated with $200 \mu \mathrm{L}$ of MAb1 $(2.2 \mathrm{mg} / \mathrm{mL}$ in distilled water) and $9 \mathrm{mg}$ of EDC. The incubation was carried out for $3 \mathrm{~h}$ with shaking at room temperature. The excess proteins were removed by centrifugation (12000 $\mathrm{rp}, 20 \mathrm{~min}$ ) and repeated 3 times. The purified conjugates were stored in PBS at $4{ }^{\circ} \mathrm{C}$.

\subsection{GNPs preparation}

GNPs were synthesized by reduction of $\mathrm{HAuCl}_{4}$ solution by trisodium citrate [27]. In brief, a $100 \mathrm{~mL}$ $\mathrm{HAuCl}_{4}$ solution was prepared in a round bottom flask. To this vigorously stirred solution, $2 \mathrm{~mL}$ of freshly prepared $1 \%$ trisodium citrate solution was added when the $\mathrm{HAuCl}_{4}$ solution was boiled. Stirring and heating was allowed to proceed for $5 \mathrm{~min}$. after the color change to red occurred. Then the colloidal gold was stirred until the solution cooled off to room temperature.

\subsection{GNPs-MAb2 conjugate preparation}

The GNPs were conjugated to anti-HBsAg monoclonal antibodies (MAb2). $200 \mu \mathrm{L}$ of MAb2 (1 mg/mL) was added to $5 \mathrm{~mL}$ of $\mathrm{pH}$-adjusted colloidal $\mathrm{Au}$ suspension ( $\mathrm{pH}$ 8.5 9.0 adjusted with $0.1 \mathrm{M} \mathrm{K}_{2} \mathrm{CO}_{3}$ ), followed by incubation for $2 \mathrm{~h}$ at room temperature. The conjugate was centrifuged at $9000 \mathrm{rpm}$ for $10 \mathrm{~min}$, and the soft sediment was resuspended in PBS (5\% BSA, pH 7.4) this step was repeated 3 times to removed the excess MAb2. The GNPs labeled MAb2 (GNPs-MAb2) were obtained and stored in PBS at $4{ }^{\circ} \mathrm{C}$.

\subsection{Detection of HBsAg}

The methodology of HBsAg detection by the novel immunoassay is shown in Fig. 1. QDs-MAb1 and GNPsMAb2 probes were used in this method. After the immuno reaction, the sandwich-type immunocomplex was formed. The procedure is described as follows: $0.05 \mathrm{~mL}$ of QDsMAb1 probes and $0.05 \mathrm{~mL}$ of GNPs-MAb2 probes were reacted simultaneously with $0.1 \mathrm{~mL}$ of HBsAg standard samples (the different concentrations of HBsAg were $0,0.5,1.5,5,15,50$, and $120 \mathrm{ng} / \mathrm{mL}$, respectively) for $30 \mathrm{~min}$ in the polystyrene tubes. Then the fluorescence quenching signal was measured by a Perkin-Elmer LS55 spectrofluorometer immediately. The excitation wavelength was at $430 \mathrm{~nm}$, and the emission spectra were recorded from 450 to $700 \mathrm{~nm}$. The excitation and emission slit widths were set to $10 \mathrm{~nm}$. Samples were put in $1 \mathrm{~cm}$ path length quartz cuvettes. The quenching signal at $570 \mathrm{~nm}$ was proportional to the amount of HBsAg present in the samples.

\section{Results and discussions}

\subsection{Characterization of QDs-MAb1}

The fluorescent intensity of QDs before and after binding with MAb1 was detected by a PerkinElmer LS

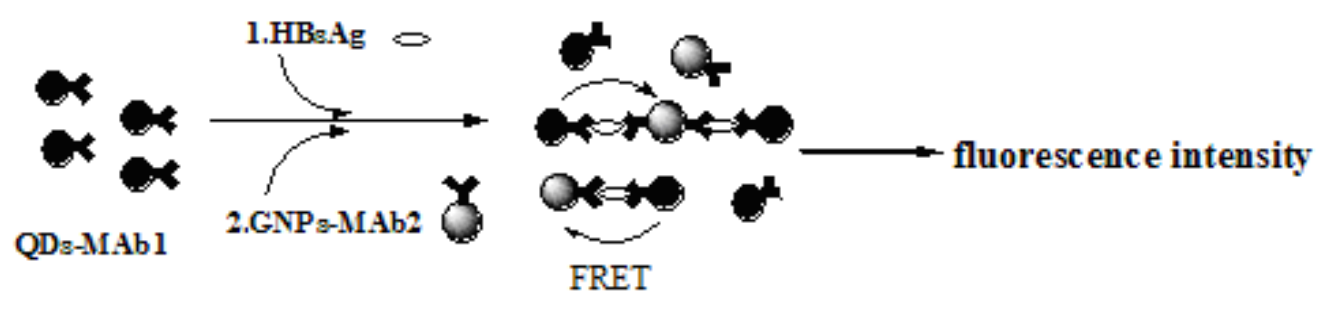

Fig. 1 Schematics of the QDs-GNPs fluorescence quenching immunoassay 


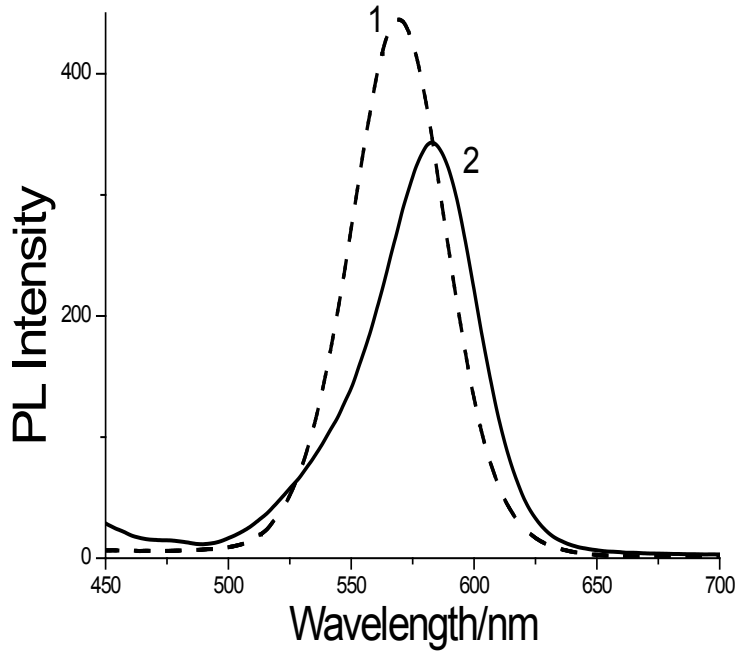

Fig. 2 Fluorescence spectra of CdTe solution before (1) and after (2) labeling with MAb1.

55 spectrofluorometer, the excitation wavelength of QDs was at $430 \mathrm{~nm}$, and the emission spectra were recorded from 450 to $700 \mathrm{~nm}$ as shown in Fig. 2. The emission maximum was at $569 \mathrm{~nm}$ for the QDs and after binding with MAb1 was at $582 \mathrm{~nm}$, the peaks moved $13 \mathrm{~nm}$. While the emission spectrum of QDs-MAb1 solution showed a red-shift in the emission peak, the intrinsic emission band width was unchanged. It was proved that the shift of the emission peak was the result of the conjugation between QDs and MAb1. Furthermore, it was also proved that the QDs-MAb1 composite wasn't agglomerated and had good dispersivity.

\subsection{Characterization of GNPs}

The properties of GNPs after binding with MAb2 was detected by UV-vis spectroscopy analysis as shown in Fig. 3 , the maximal absorbance $\left(\lambda_{\max }\right)$ was at $522 \mathrm{~nm}$ for the GNPs and after binding with MAb2, $\lambda_{\max }$ values shifted to longer wavelength at $536 \mathrm{~nm}$. The UV-vis spectra proved the conjugation between GNPs and proteins.

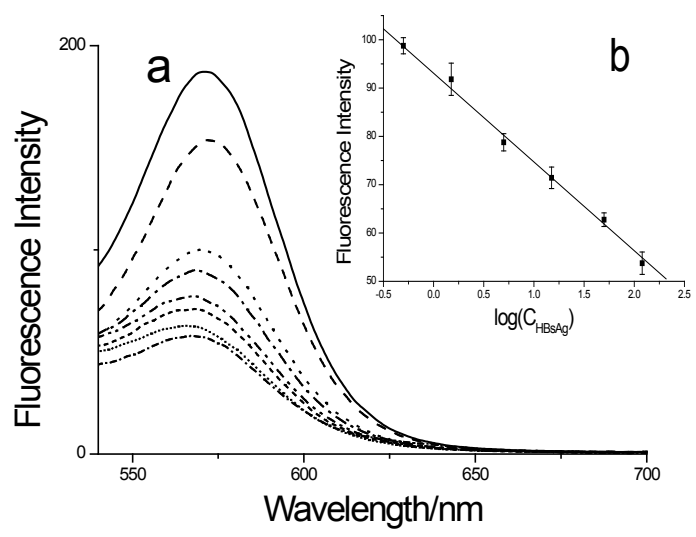

Fig. 4 (a) Effect of GNPs-MAb2 probes on the fluorescence spectra of FITC. HBsAg concentration increased from top to bottom: 0, 0.5, 1.5, 5, 15,50 , and $120 \mathrm{ng} / \mathrm{mL}$, and the solid line was the control of QDs-MAb1 without GNPs-MAb2 probes. (b) Plots of the fluorescence intensity at $570 \mathrm{~nm}$ when HBsAg concentration increased.

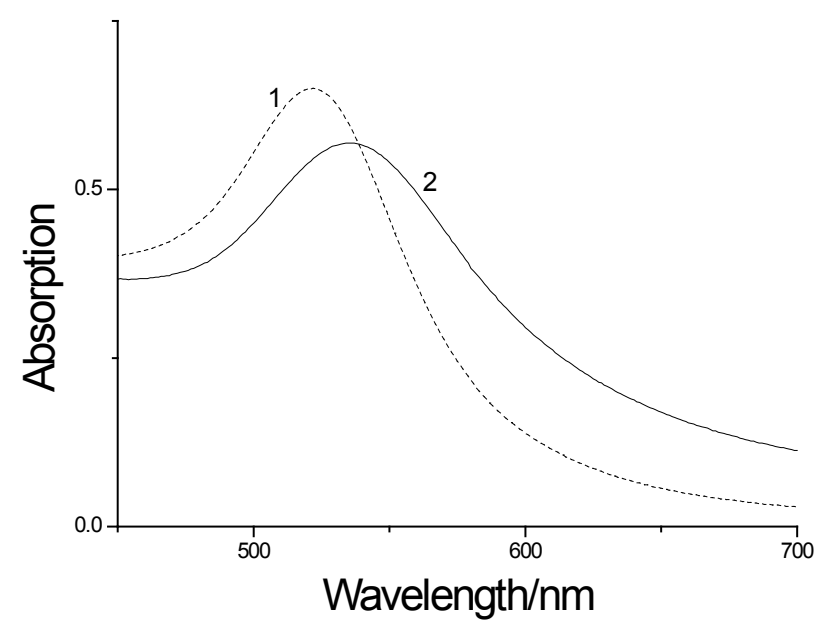

Fig. 3 UV-vis spectra of GNPs samples: (1) GNPs; (2) GNPs-MAb2

\subsection{Detection of HBsAg}

The principle of the novel immunoassay based on the PL quenching of QDs by GNPs was described in Fig. 1. To examine whether FRET between QDsMAb1 and GNPs-MAb2 is specific and applicable to the immunoassay, we monitored the changes in the PL spectra of reaction liquids at different concentration of HBsAg by using a photoluminescence spectrometer as shown in Fig. 4. The fluorescence intensity of reaction liquids was quenched over $50 \%$ (Fig. 4a) and decreased gradually at $570 \mathrm{~nm}$ as the concentration of $\mathrm{HBsAg}$ increased from 0 to $120 \mathrm{ng} / \mathrm{mL}$ (Fig. $4 \mathrm{~b}$ ). On the other hand, when the QDs-MAb1 were replaced by QDs, the fluorescence intensity of QDs was quenched about $20 \%$ and the quenching percentage was almost unchanged during the concentration of HBsAg increased from 0 to $120 \mathrm{ng} / \mathrm{mL}$ as shown in Fig. 5. It was evident that FRET occurs when QDs and GNPs are within a short distance by specific interaction between QDs-MAb1 and GNPsMAb2 with HBsAg (antigen), which induces an efficient

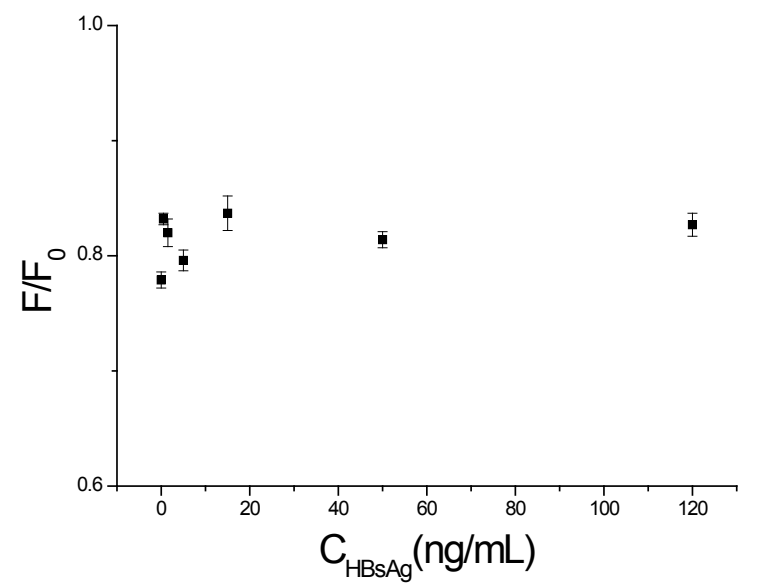

Fig. 5 The plot of GNPs-MAb2 probes quenching QDs at different concentrations of HBsAg (F: fluorescence intensity of QDs with the presence of GNPs-MAb2; F0: fluorescence intensity of QDs without the presence of GNPs-MAb2) 
energy transfer from the donor (QDs-MAb1) to the acceptor (GNPs-MAb2).

On the basis of the above results, we attempted an assay for HBsAg as a model protein. After the immuno reaction, the sandwich-type immunocomplex of the QDsMAb1 and GNPs-MAb2 was increased as the concentration of HBsAg increased. Fig. 4a represents that FRET happened between CdTe nanocrystals and gold nanoparticles in the course of immunoreaction. The PL intensity at $570 \mathrm{~nm}$ is negative linear proportional to HBsAg concentration logarithm (Fig. 4b). The limit of detection was about $0.928 \mathrm{ng} / \mathrm{mL}$ under our experimental condition, which was calculated by the interpolation of the mean plus two standard deviations of 10 replicates of the zero standards.

\section{Conclusions}

Water soluble quantum dots such as CdSe, CdTe nanocrystals, etc. have been being explored application in biological analysis and cell imaging. Herein we investigated a new FRET immunoassay based on GNPs and QDs. The energy of quantum dots was transferred to gold nanoparticles as they were within a short distance by the sandwich-type immuno reactions. We have demonstrated that FRET between QDs and GNPs can be used for determination of the antigen and the whole procedure was very simple. Herein we attempted an assay for $\mathrm{HBsAg}$ as a model protein; the assay is general for the detection of the target molecules with known matched antibodies. Therefore, the new method could be used widely in immunoassays.

\section{Acknowledgements}

This work is supported by the National Key Basic Research Program (973 Project) (No. 2011CB933100), National Natural Scientific Fund (No.81225010, 31100717), 863 project of China (2012AA022703), Shanghai Science and Technology Fund (No.10XD1406100, and 13NM1401500), Shanghai Jiao Tong University Innovation Fund for Postgraduates (No. AE340011).

\section{References}

1 Stryer L. Fluorescence energy transfer as a spectroscopic ruler. Annu. ReV. Biochem., 1978, 47, 819-846.

2 Fairclough R.H., Cantor C.H. The use of singlet-singlet energy transfer to study macromolecular assemblies. Methods Enzymol., 1978, 48, 347-379.

3 Wu P.G,. Brand L. Resonance energy transfer: methods and applications. Anal. Biochem., 1994, 218, 1-13.

4 Meer B., Coker. G., Chen. S. Y.S, Resonance Energy Transfer: Theory and Data, Cambridge: VCH : New York, 1994.

5 Selvin P.R, Fluorescence resonance energy transfer. Methods Enzymol., 1995, 246, 300-334.

6 Schobel U., Egelhaaf H.J., Brecht A., Oelkrug D., Gauglitz G. New donor-acceptor pair for fluorescent immunoassays by energy transfer. Bioconjugate Chem., 1999, 10, 1107-1114.

7 Kagan C.R., Murray C.B., Nirmal M., Bawendi M.G. Electronic energy transfer in CdSe quantum dot solids. Phys. ReV. Lett., 1996, 76, 1517-1520.

8 Kagan C.R., Murray C.B., Bawendi, M. G, Long-range resonance transfer of electronic excitations in close-packed CdSe quantumdot solids. Phys. ReV. B., 1996, 54, 8633-8643.

9 Finlayson C.E., Ginger D.S., Greenham N.C. Enhanced Förster energy transfer in organic/inorganic bilayer optical microcavities.
Chem. Phys. Lett., 2001, 338, 83-87.

10 Mamedova N.N., Kotov N.A., Rogach A.L., Studer J. AlbuminCdTe nanoparticle bioconjugates: preparation, structure, and interunit energy transfer with antenna effect. Nano Lett., 2001, 1, 281-286.

11 Ruan J., Shen J., Song H., Ji J.J., Wang K., Cui D.X., Wang Z. Viability and pluripotency studying of human embryo stem cells labeled with quantum dots. Nano Biomed. Eng. 2010, 2(4),245251.

12 Cui D.X., Zhang H., Sheng J., Wang Z., Toru A, He R., Tetsuya O., Gao F., Cho H.S., Huth C., Hu H.Y., Pauletti G.M., Shi D.L. Effects of $\mathrm{CdSe} / \mathrm{ZnS}$ quantum dots covered multi-walled carbon nanotubes on murine embryonicstem cells. Nano Biomed. Eng. 2010, 2(4), 236-244.

13 Bruchez M., Jr, Moronne M., Gin P., Weiss S., Alivisatos A.P. Semiconductor nanocrystals as fluorescent biological labels. Science, 1998, 281, 2013-2016.

14 Chan W.C.W., Nie S. Quantum dot bioconjugates for ultrasensitive nonisotopic detection. Science, 1998, 281, 2016-2018.

15 Mattoussi H., Mauro J.M., Goldman E.R., Anderson G.P, Sundar V.C., Mikulec F.V., Bawendi M.G. Self-assembly of CdSe-ZnS quantum dot bioconjugates using an engineered recombinant protein. J. Am. Chem. Soc., 2000, 122, 12142-12150.

16 Pathak S., Choi S.K., Arnheim N., Thompson M.E. Hydroxylated quantum dots as luminescent probes for in situ hybridization. J. Am. Chem. Soc., 2001, 123, 4103-4104.

17 Willard D.M., Carillo L.L., Jung J., Van Orden A. CdSe-ZnS quantum dots as resonance energy transfer donor in a model proteinprotein binding assay. Nano Lett., 2001, 1, 469-474.

18 Sonay A.Y., Keseroğlu K., Culha M. 2D Gold Nanoparticle StructuresEngineered Through DNA Tiles for Delivery, Therapy. Nano Biomed. Eng. 2012, 4(1), 17-22.

19 Zhi X., Zhang X., Huang P., Cui D.X. Effects of Chiral Gold Nanoflowers on Polymerase Chain Reaction. Nano Biomed. Eng. 2011, 3(4), 256-259.

20 Cui D.X., Ozkan C.S., Tian F.R., Kong Y. Delivery of Gold Nanoparticles Inside Carbon Nanotubes by Oligonucleotides. Nano Biomed. Eng. 2011, 3(4), 243-248

21 Abel B., Akinsule A., Andrews C., Aslan K. Plasmon-Enhanced Enzymatic Reactions: A Study of Nanoparticle-Enzyme Distance-and Nanoparticle Loading-Dependent Enzymatic Activity. Nano Biomed. Eng. 2011, 3(3), 184-191

22 Dulkeith E., Morteani, A.C., Niedereichholz T.A., Klar T.A., Feldmann J. Fluorescence quenching of dye molecules near gold nanoparticles: radiative and nonradiative effects. Phys. ReV. Lett., 2002, 89, 203002.

23 Wargnier R., Baranow. A.V., Maslov V.G., Stsiapura V., Artemyev M., Pluot M., Sukhanova A., Nabiev I. Energy transfer in aqueous solutions of oppositely charged $\mathrm{CdSe} / \mathrm{ZnS}$ core/shell quantum dots and in quantum dot-nanogold assemblies. Nano Lett., 2004, 4, 451-457.

24 Ao L.M., Gao F., Pan B.F., He R., Cui D.X. Fluoroimmunoassay for antigen based on fluorescence quenching signal of gold nanoparticles. Anal. Chem., 2006; 78: 1104-1106.

25 Cui D.X., Pan B.F., Zhang H., Gao F., Wu R., Wang J.P., He R., Asahi T. Self-Assembly of Quantum Dots and Carbon Nanotubes for Ultrasensitive DNA and Antigen Detection. Anal. Chem., 2008; 80: 7996-8001.

26 Goldman E.R., Balighian E.D., Mattoussi H., Kuno M.K., Mauro J.M., Tran P.T., Anderson G.P. Avidin: a natural bridge for quantum dot-antibody conjugates. J. Am. Chem. Soc., 2002, 124, 6378-6382.

27 Zhu T., Vasilev K., Kreiter M., Mittler S., Knoll W. Surface modification of citrate-reduced colloidal gold nanoparticles with 2-mercaptosuccinic acid. Langmuir. 2003, 19, 9518-9525.

Copyright:(c) 2013 C. Wang et al. This is an open-access article distributed under the terms of the Creative Commons Attribution License, which permits unrestricted use, distribution, and reproduction in any medium, provided the original author and source are credited. 